EPJ Web of Conferences 32, 04019 (2012)

DOI: 10.1051/epjconf/20123204019

(C) Owned by the authors, published by EDP Sciences, 2012

\title{
A study on the output beam profile of a ring resonator type diplexer
}

\author{
Y.Oda $^{1}$, S. Sugawara ${ }^{2}$, K. Atsumi ${ }^{2}$, T. Yamaguchi ${ }^{2}$, M. Saigusa ${ }^{2}$, K. Sakamoto ${ }^{1}$ \\ ${ }^{1}$ Japan Atomic Energy Agency \\ ${ }^{2}$ Ibaraki University
}

\begin{abstract}
A ring resonator type diplexer was tested for fast switching device for ECCD system. The ring resonator was composed by circular corrugated waveguides with 63.5 mm diameter. Four in/output ports were separated from the resonator by half mirrors. The resonance frequency and its interval were designed as $170 \mathrm{GHz}$ and $460 \mathrm{MHz}$, respectively. The mock-up diplexer which utilizes slotted planes with $2 \mathrm{~mm}$ period or sapphire disk with $1 \mathrm{~mm}$ thickness as half mirrors were examined at using $170 \mathrm{GHz}-$ band low power oscillator. RF beam with $95 \%$ of the fundamental mode $\left(\mathrm{LP}_{01}\right.$ mode) was provided into the input port of the diplexer using a mode generator. Beam profiles from output ports were measured and mode contents in output ports were deduced. When the slotted mirrors were utilized, the $\mathrm{LP}_{01}$ mode purity was decreased as $85 \%$ at the resonance frequency, $170 \mathrm{GHz}$. This was because higher order modes were induced by the higher order diffraction beams from the slotted half mirror. On the other hand, mode purity was increased higher than $98 \%$ at $170 \mathrm{GHz}$ when sapphire mirrors were utilized.
\end{abstract}

\section{Introduction}

Recently, high-power millimetre wave diplexers which utilize the RF resonance cavity have been developed for the electron cyclotron current drive (ECCD) in fusion devices. These diplexers are expected to operate as a directional switch for control of instability in fusion plasma. A neoclassical tearing mode (NTM) is a resistive MHD instability driven by plasma pressure gradient in tokamak plasma. To control NTM, ECCD RF power should provide to drive local current in a magnetic island tracking RF injection position into the rotating O-point is necessary [1]. Recently ECCD with pulse modulated gyrotron operation at duty of $50 \%$ have been done to drive current. For improving a stabilizing efficiency of NTM, the fast directional switch had been developed $[2,3]$. It makes the duty of ECCD system to $100 \%$ by switching beam direction for tracking the rotating O-point of a magnetic island of NTM. Since switching of the diplexer with resonance cavity is operated by frequency modulation of gyrotorn, the switching speed can be increased up to $\mathrm{kHz}$ order.

The new type diplexer as a fast switching device of high power millimeter wave was proposed. The diplexer was designed for wide frequency band operation utilizing half mirrors and it had been simulated with finite difference time domain (FDTD) method $[4,5]$. In this paper, the mock-up diplexer for corrugated waveguide system was examined. The mode purity of the output beam from the diplexer with was measured and mode conversion in the diplexer was discussed. 


\section{Experimental Apparatus}

\subsection{Mock-up model of waveguide ring resonator type diplexer}

The ring resonator, as shown in Fig.1, was composed by circular corrugated waveguides with $63.5 \mathrm{~mm}$ diameter. Four in/output ports were separated from the resonator by half mirrors. The mock-up diplexer utilizes slotted plates or the sapphire disk as half mirrors. The resonant ring length was settled about $650 \mathrm{~mm}$. The resonance frequency interval was designed as $460 \mathrm{MHz}$. The body of the diplexer was made of aluminum alloy and a pair of reflector panel was made of oxygen free copper. The slotted metal half mirrors made of aluminum alloy are installed in the resonant ring. The thickness of the slotted plate is $1.7 \mathrm{~mm}$. The period and the width of slots are $2.0 \mathrm{~mm}$ and $1.0 \mathrm{~mm}$, respectively. The slot direction is parallel to the incident plane. The electric field of $\mathrm{HE}_{11}$ mode is vertical of the incident plane for reducing Ohmic loss of mitre-bends and suppressing counter rotating modes in a ring resonator. The diplexer was designed to capable replacing the half mirrors. The sapphire disk with $1 \mathrm{~mm}$ thickness was also utilized for half mirrors and tested. The power reflection coefficient of slotted metal half mirror and sapphire mirror was evaluated to be 0.42 and 0.9 at a frequency of $170 \mathrm{GHz}$.

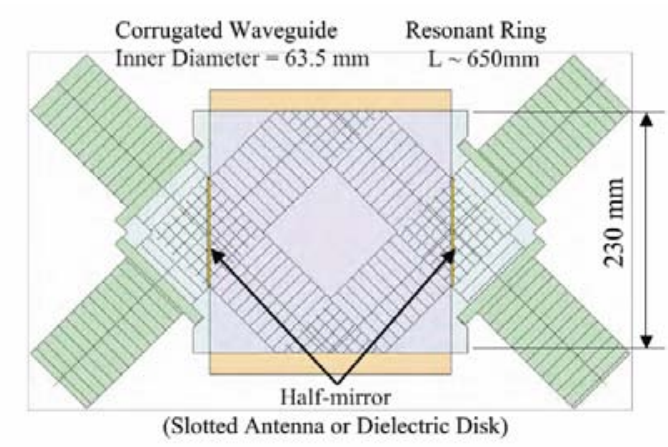

Fig. 1. Schematic figure of diplexer configuration.

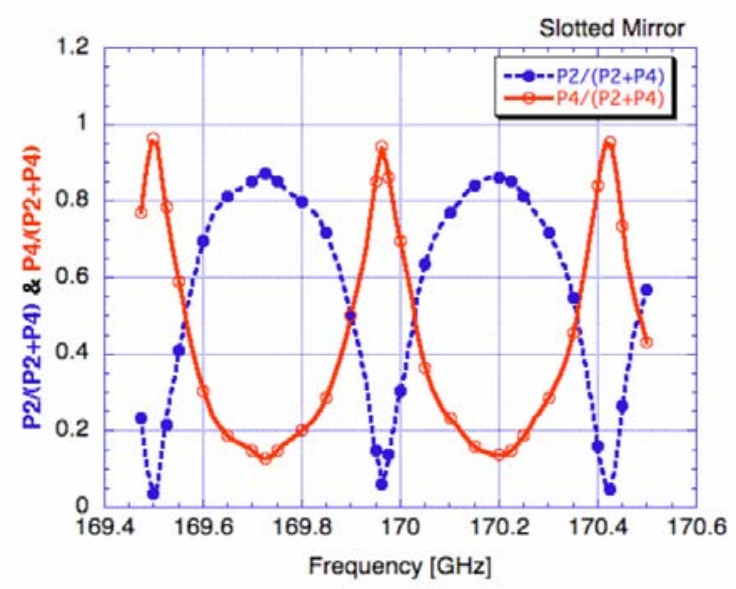

Fig. 2. Dependence of output power from diplexer with slotted mirror on operation frequency.[6] 
When the input RF from input port (p1) sets non-resonant frequency, most power is reflected at the half mirror and transmits to an output port (p2). When the input RF sets the resonant frequency, input power propagates through the resonator and transmits to another output port (p4). The dependence of output power on frequency for the diplexer using slotted plate was measured in ref.6 as shown in Fig.2. The resonance frequency was found around $170.0 \mathrm{GHz}$.

\subsection{Low Power Measurement Facility}

The mock-up diplexer was examined at using $170 \mathrm{GHz}$-band low power oscillator. Beam profiles from $\mathrm{p} 2$ and $\mathrm{p} 4$ were measured using the heterodyne detecting system at RF frequency of 170.0 $\mathrm{GHz}-170.5 \mathrm{GHz}$. Figure 3 shows the measurement setup for the diplexer. The RF power was injected into $\mathrm{p} 1$ port from $\mathrm{HE}_{11}$ mode generator. The mode purity of $\mathrm{HE}_{11}$ generator output beam included $95 \%$ of the fundamental mode, $\mathrm{LP}_{01}\left(\mathrm{HE}_{11}\right)$ mode, and $2 \%$ of $\mathrm{LP}_{02}$. The amplitude profiles of radiated beams from $\mathrm{p} 2$ and $\mathrm{p} 4$ were measured using a waveguide antenna scanned in the area of $100 \times 100 \mathrm{~mm}$ at $100,200,300,400$, and $500 \mathrm{~mm}$ from the waveguide edge using the 2-D scan stage. Mode contents were deduced by phase retrieved method using measured profiles. Then the diplexer with the sapphire half mirrors was tested as same.

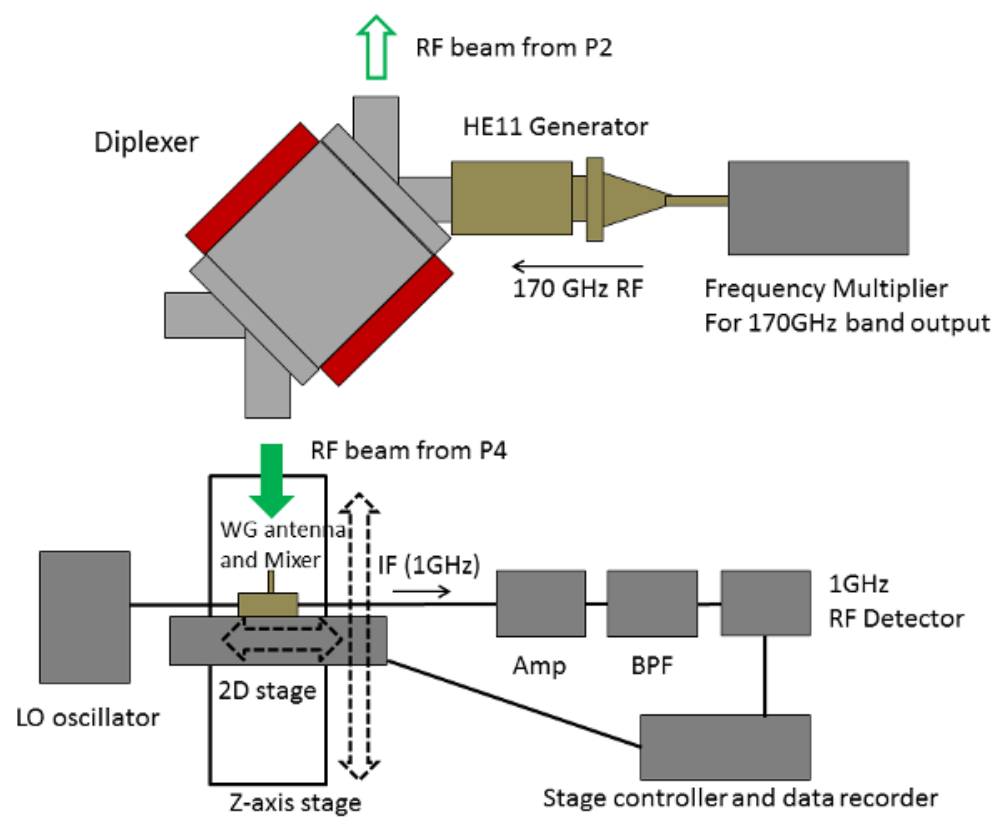

Fig. 3. Configuration of low power oscillator and detector system used for diplexer experiment.

\section{Experimental Results}

\subsection{Slotted plate mirror}

The measured mode contents from $\mathrm{p} 2$ and $\mathrm{p} 4$ output of the diplexer with slotted metal plate mirror were plotted on Fig.4. The fundamental transmission mode $\left(\mathrm{LP}_{01}\left(\mathrm{HE}_{11}\right)\right.$ mode) purity in output power from $\mathrm{p} 2$ was $93 \%$ at $170.1 \mathrm{GHz}$ operation. The mode purity of $\mathrm{LP}_{01}$ mode was 
degraded with increase of operation frequency and it was decreased to $91 \%$ at $170.3 \mathrm{GHz}$. On the other hand, the $\mathrm{LP}_{01}$ mode purity of output power from $\mathrm{p} 4$ at resonance frequency was only $85 \%$. The output power from both ports included some higher order modes and higher order modes from p2 were increased with operation frequency. The dominant higher order modes from $\mathrm{p} 2$ were $2-4 \%$ of $\mathrm{LP}_{11}$ (odd) mode and 1-2\% of LP02 mode. At resonance frequency, 5-9\% of $\mathrm{LP}_{11}(\mathrm{odd})$ mode and $2-3 \%$ of $\mathrm{LP}_{02}$ modes were detected and $\mathrm{LP}_{11}$ (odd) mode was nearly double of content from $\mathrm{p} 2$. These higher order modes were induced by the higher order diffraction beams from the slotted half mirror. At resonant frequency, higher order modes generated by the diffraction beams were multiplied by the resonance which caused the large mode conversion. Then mode purity of p4 output was far worse than p2 output. Though the switching operation was successfully demonstrated, further optimization of slotted half mirror is required to reduce mode conversion loss in the diplexer.

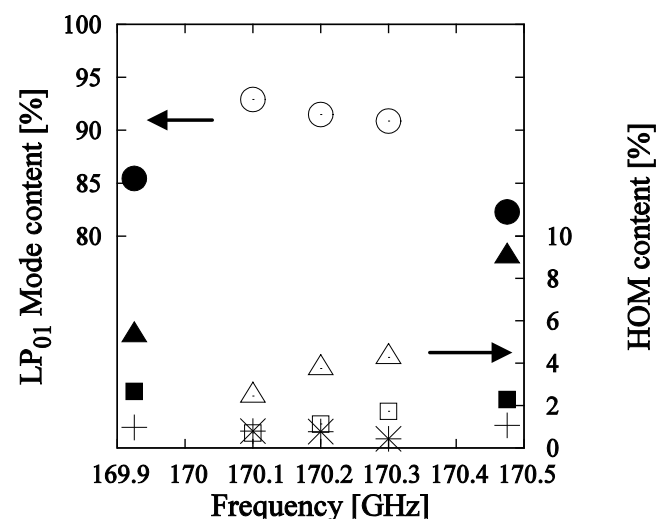

Fig. 4. Dependence of mode contents from output on operation frequency for the diplexer with slotted mirrors. Mode content of output power from $\mathrm{p} 4\left(\mathbf{O}: \mathrm{LP}_{01}\right.$ mode, $\boldsymbol{\Delta}: \mathrm{LP}_{11}$ (odd) mode, $\mathbf{\mathbf { a }}: \mathrm{LP}_{02}$ mode, $+: \mathrm{LP}_{11}$ (even) mode) and mode content of output power from $\mathrm{p} 2\left(\bigcirc: \mathrm{LP}_{01}\right.$ mode, $\Delta: \mathrm{LP}_{11}$ (odd) mode, $\square: \mathrm{LP}_{02}$ mode, $\times: \mathrm{LP}_{11}($ even) mode)

\subsection{Sapphire mirror}

The measured mode contents from p2 and p4 output of the diplexer with sapphire disk were plotted on Fig.5. For p2 output beam, $\mathrm{LP}_{01}$ mode purity was $95 \%$ at $170.1 \mathrm{GHz}$ operation and $94 \%$ at $170.3 \mathrm{GHz}$ which were similar content with slotted mirror. The $\mathrm{LP}_{01}$ mode purity of output power from $\mathrm{p} 4$ at resonance frequency was higher than $98 \%$ which was even higher content than input content. The dominant higher order modes from $\mathrm{p} 2$ were $2-3 \%$ of $\mathrm{LP}_{02}$ mode and $1-2 \%$ of $\mathrm{LP}_{11}$ (odd) mode. At resonance frequency, contents of $\mathrm{LP}_{11}$ and $\mathrm{LP}_{02}$ modes were less than $1 \%$. This measurement result indicates that the diplexer can be utilized for a mode filter system at resonance frequency operation. In contrast with slotted mirror, the sapphire mirror does not generate the higher order diffraction beams. Then the resonation of the fundament mode was excited in the ring which could reduce unwanted higher order modes included in input beam. 


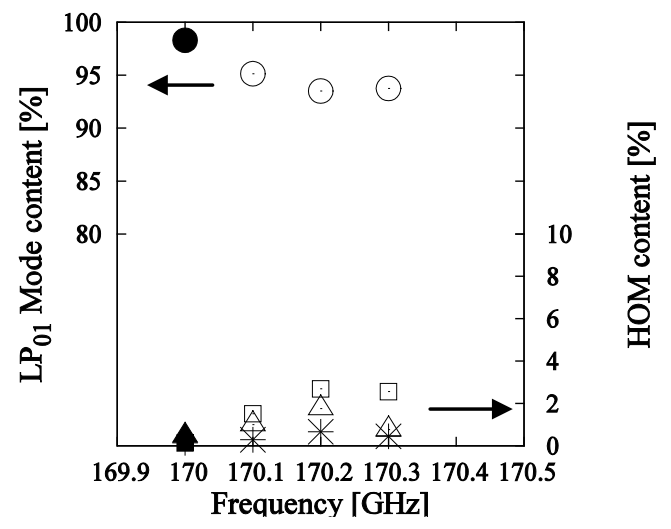

Fig. 5. Dependence of mode contents from output on operation frequency for the diplexer with sapphire mirrors. Mode content of output power from $\mathrm{p} 4\left(\mathbf{O}: \mathrm{LP}_{01}\right.$ mode, $\boldsymbol{\Delta}: \mathrm{LP}_{11}$ (odd) mode, $\mathbf{\square}: \mathrm{LP}_{02}$ mode, $+: \mathrm{LP}_{11}$ (even) mode) and mode content of output power from $\mathrm{p} 2\left(\bigcirc: \mathrm{LP}_{01}\right.$ mode, $\Delta: \mathrm{LP}_{11}$ (odd) mode, $\square: \mathrm{LP}_{02}$ mode, $\times: \mathrm{LP}_{11}($ even $)$ mode)

\section{Summary}

Mode content of output beam from diplexer was measured. The diplexer with slotted mirror was measured and the $\mathrm{LP}_{01}$ mode purity from $\mathrm{p} 2$ was varied with frequency and mode purity from $\mathrm{p} 4$ was $85 \%$ and $\mathrm{LP}_{11}$ mode was significantly increased. Higher order modes were induced by the higher order diffraction beams from the slotted half mirror.

The diplexer with sapphire mirror was also measured and $\mathrm{LP}_{01}$ mode of output beam from $\mathrm{p} 4$ was found as $98 \%$ which was higher than input beam. Since the sapphire mirror does not generate the higher order diffraction beam, the resonance of the fundamental mode was excited and unwanted higher order modes were reduced.

\section{References}

1. H. Zohm, G. Gantenbein, G. Giruzzi, S. Gunter, F. Leuterer, M. Maraschek, et al., Nucl. Fusion 39, 577 (1999)

2. M.I. Petelin, "Quasi-optics in high-power millimeter-wave systems,” AIP Conf. Proc 691 (2003) p. 251.

3. W. Kasparek, et al., Fusion Sci. Technol. 52 (2007) p. 281.

4. M. Saigusa, et al., "Fast switching device of high power millimeter wave in ECCD system" Proc. of $13^{\text {th }}$ AMPERE Toulouse (2011), p. 285.

5. K. Atsumi, et al., "Research and Development of High Power Wideband Diplexer for ECCD System" Plasma Conference 2011, Kanazawa, 23P099-P, (2011).

6. M. Saigusa, et al., "Proposal of New Type Diplexer for ECCD System," 21st International Toki Conference (2011) 